Case Report

\title{
Double Ventricular Responses Leading to Reversible Cardiomyopathy as Late Complication after Slow-Pathway Ablation
}

\author{
Umut Celikyurt, Meinrad Gawaz, Juergen Schreieck, and Peter Seizer \\ Medizinische Klinik III, Kardiologie und Kreislauferkrankungen, Eberhard Karls Universität, Tübingen, Germany \\ Correspondence should be addressed to Peter Seizer; peter.seizer@med.uni-tuebingen.de
}

Received 15 May 2015; Revised 14 September 2015; Accepted 16 September 2015

Academic Editor: Gianluca Di Bella

Copyright (C) 2015 Umut Celikyurt et al. This is an open access article distributed under the Creative Commons Attribution License, which permits unrestricted use, distribution, and reproduction in any medium, provided the original work is properly cited.

Double ventricular response is a rare arrhythmia that results from simultaneous antegrade conduction over the fast and slow pathways of AV node. Double ventricular responses may lead to arrhythmia-related cardiomyopathy. As far as we know, there is not any reported reversible cardiomyopathy development that resulted from double ventricular responses to one atrial impulse after slow pathway ablation. We report a unique case of double ventricular response cardiomyopathy that has been developed 5 years after slow pathway ablation.

\section{Introduction}

Double ventricular response is a rare arrhythmia that results from simultaneous antegrade conduction over the fast and slow pathways of AV node. Slow-pathway conduction must be slow enough to allow his-purkinje system to recover excitability after depolarization by the first excitation over the fast pathway. Double ventricular responses may lead to tachycardia-related cardiomyopathy. We report a unique case of double ventricular response cardiomyopathy that has been developed 5 years after slow-pathway ablation.

\section{Case Report}

A 69-year-old patient admitted to our ambulance with severe dyspnea. Five years ago a slow-pathway cryoablation due to typical atrioventricular nodal reentry tachycardia was performed. Transthoracic echocardiography performed at admission revealed severely reduced left ventricular ejection fraction (LVEF) (30\%), which was reported normal 5 years ago. In the next step, haemodynamic relevant stenoses were excluded in coronary angiography. Cardiac MRI confirmed severely reduced LVEF without the presence of myocardial fibrosis. Electrocardiography (ECG) revealed an uncommon finding of two ventricular beats after one atrial beat
(Figure 1(a)). An electrophysiological study was performed due to this uncommon ECG. ECG revealed a double ventricular excitation due to a dual AV-node conduction capacity (Figures 1(b) and 1(c)). Slow-pathway ablation was performed again with radiofrequency using a solid tip catheter $(4 \mathrm{~mm}$, 30 watt, Figures 2(a) and 2(b)). Junctional beats appeared 15 seconds after start of ablation, the second ventricular excitation terminated, and a stable sinus rhythm was established. The heart rate dropped from $100 \mathrm{bpm}$ (double ventricular response) to about $50 \mathrm{bpm}$ in telemetric monitoring and ECG revealed normal sinus rhythm (Figure 2(c)). Interestingly, the LVEF was improved to $40 \%$ after two months. After 12 months LVEF was completely normalized and left ventricular end-diastolic diameter was decreased from $59 \mathrm{~mm}$ to $53 \mathrm{~mm}$.

To the best of our knowledge, this is the first report of reversible cardiomyopathy due to double ventricular response to one atrial impulse that developed as a late complication of slow-pathway cryoablation.

\section{Discussion}

Atrioventricular nodal reentrant tachycardia (AVNRT) is one of the most common sustained supraventricular arrhythmia. Catheter ablation of the slow pathway is treatment of choice for patients with recurrent drug-refractory AVNRT [1]. The 


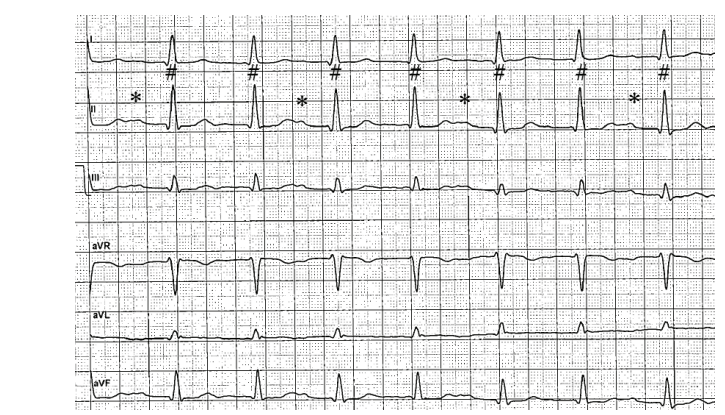

(a)
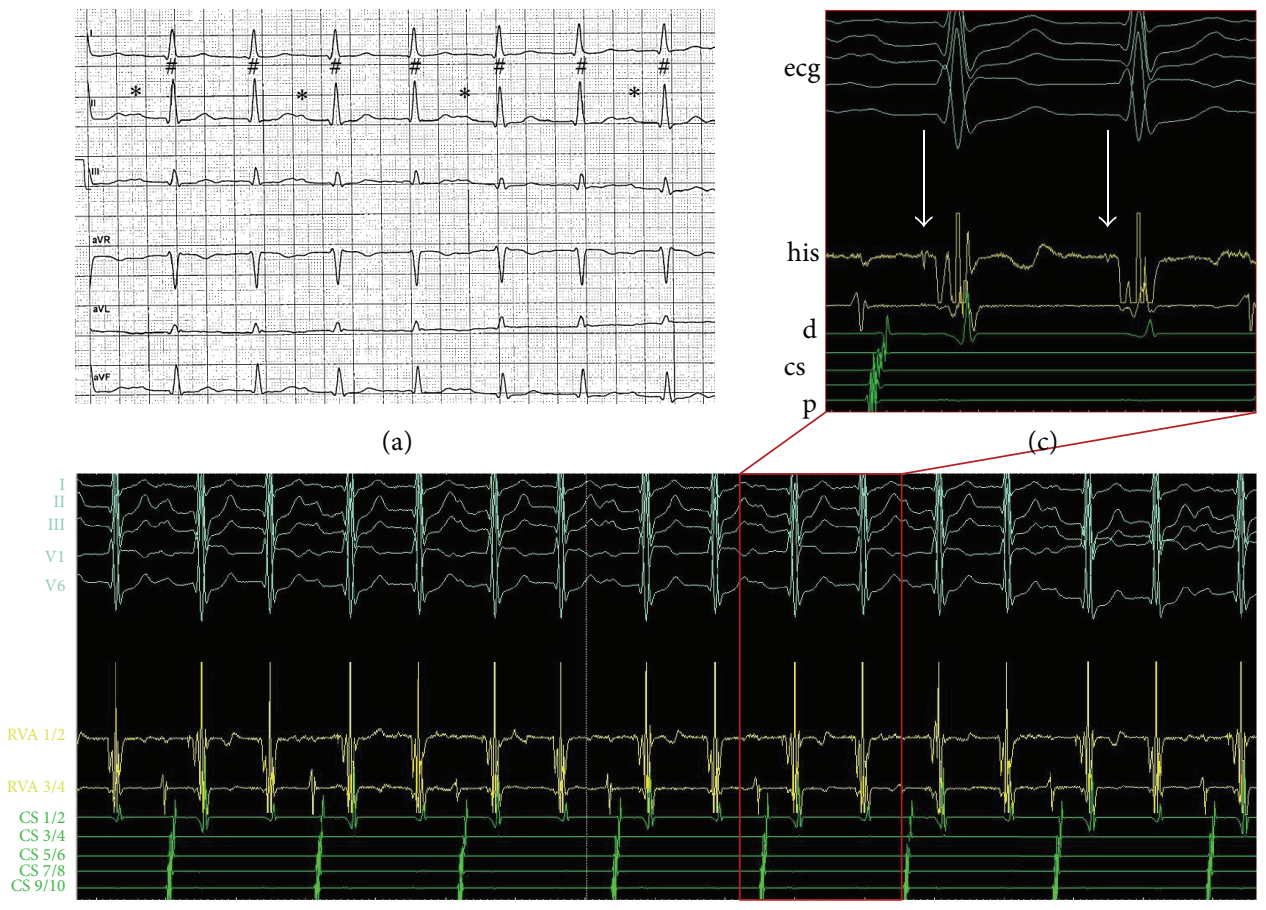

(b)

Figure 1: (a) ECG showing two ventricular beats (\#) after one atrial beat (*); (b) intracardiac electrocardiogram showing two ventricular beats after one atrial beat; (c) intracardiac electrocardiogram revealed his potential before each ventricular signal (see arrows). ECG: electrocardiography.

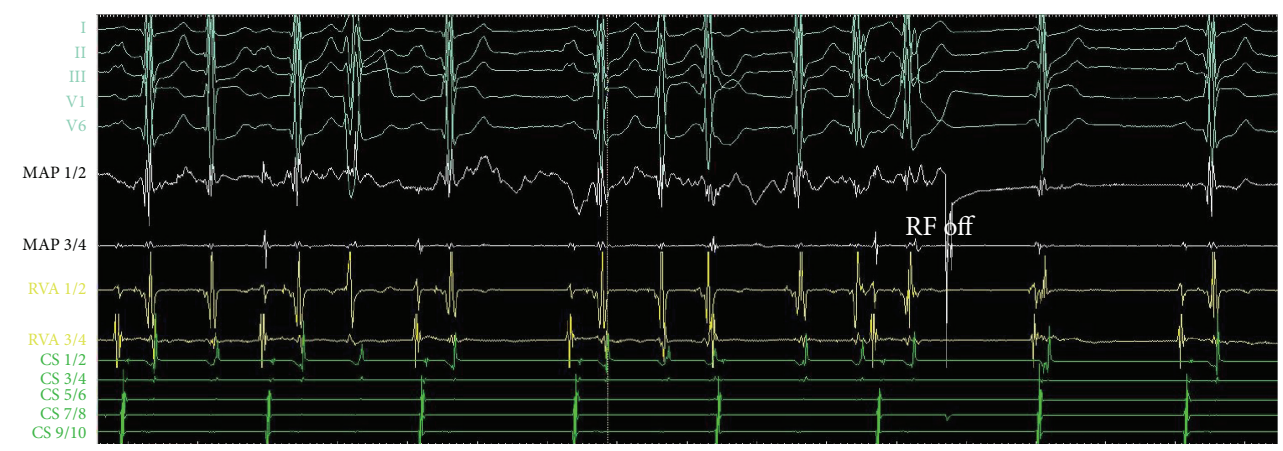

(a)

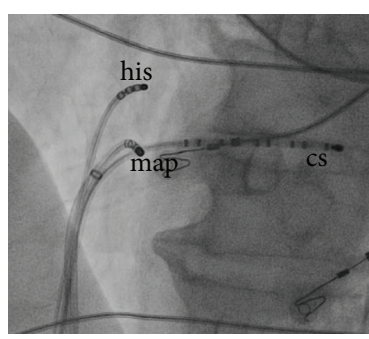

(b)

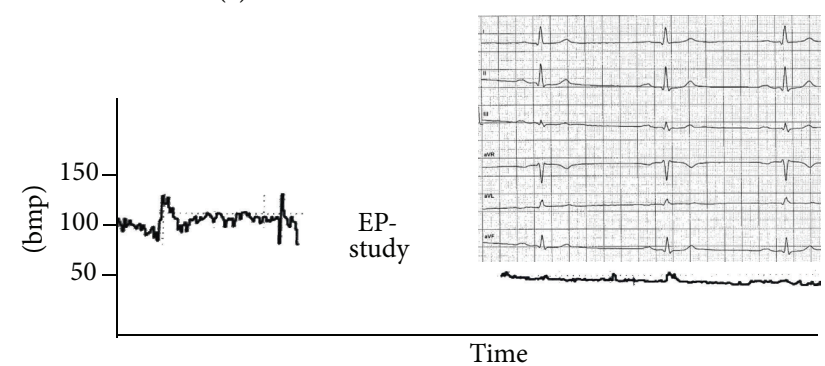

(c)

FIGURE 2: (a) Intracardiac electrocardiogram showing disappearance of the arrhythmia during ablation therapy with radiofrequency (RF). (b) Fluoroscopy $\left(45^{\circ} \mathrm{LAO}\right)$ showing ablation catheter in slow-pathway position during ablation. (c) Drop in heart rate after successful ablation in continuous heart rate monitoring and postablation ECG showing normal sinus rhythm. ECG: electrocardiography, CS: coronary sinus, bmp: beats per minute, and RF: radiofrequency. 
risk of periprocedural atrioventricular block is a known complication and less than $1 \%$ of patients treated with radiofrequency ablation have been reported [2]. As far as we know, there is not any reported reversible cardiomyopathy development that resulted from double ventricular responses to one atrial impulse after slow-pathway ablation.

Double ventricular response is a rare arrhythmia that results from simultaneous antegrade conduction over the fast and slow pathways of AV node [3]. Slow-pathway conduction must be slow enough to allow his-purkinje system to recover excitability after depolarization by the first excitation over the fast pathway. Also, there is unidirectional retrograde block in fast and slow pathways [4].

The presence of his electrograms before each QRS complex and a relatively stable interval between the fast- and slowpathway his electrograms during electrophysiological study supports the concept. However, simple junctional extrabeats as bigeminus cannot fully be excluded. The termination of the tachycardia with slow-pathway ablation is the treatment of choice.

Although double ventricular responses and tachycardiarelated cardiomyopathy that reverses with slow-pathway ablation has been described in the literature, our case is unique in which double ventricular responses and related cardiomyopathy occurred 5 years after slow-pathway ablation.

It is known that there may be late recovery of conduction after successful ablation [5]. In our patient, the delayed development of dual ventricular responses to one atrial stimulus after slow-pathway ablation may result from partial restoration of electrophysiological function at slow pathway.

The normalization of LV function after ablation strongly supports that cardiomyopathy was related to the dual ventricular responses.

\section{Conflict of Interests}

The authors declare that there is no conflict of interests regarding the publication of this paper.

\section{Acknowledgments}

Authors have been supported, in part, by grants from the Deutsche Forschungsgemeinschaft (Transregio SFB19 and the Klinische Forschergruppe KFO274) and the Tuebingen Platelet Investigative Consortium (TuePIC).

\section{References}

[1] P. Santangeli, R. Proietti, L. Di Biase, R. Bai, and A. Natale, "Cryoablation versus radiofrequency ablation of atrioventricular nodal reentrant tachycardia," Journal of Interventional Cardiac Electrophysiology, vol. 39, no. 2, pp. 111-119, 2014.

[2] H. L. Estner, G. Ndrepepa, J. Dong et al., "Acute and long-term results of slow pathway ablation in patients with atrioventricular nodal reentrant tachycardia-an analysis of the predictive factors for arrhythmia recurrence," Pacing and Clinical Electrophysiology, vol. 28, no. 2, pp. 102-110, 2005.
[3] M. Mansour, N. Marrouche, J. Ruskin, A. Natale, and D. Keane, "Incessant nonreentrant tachycardia due to simultaneous conduction over dual atrioventricular nodal pathways mimicking atrial fibrillation in patients referred for pulmonary vein isolation," Journal of Cardiovascular Electrophysiology, vol. 14, no. 7, pp. 752-755, 2003.

[4] B. C. Mani and B. B. Pavri, "Dual atrioventricular nodal pathways physiology: a review of relevant anatomy, electrophysiology, and electrocardiographic manifestations," Indian Pacing and Electrophysiology Journal, vol. 14, no. 1, pp. 12-25, 2014.

[5] S. Nath, J. G. Whayne, S. Kaul, N. C. Goodman, A. R. Jayaweera, and D. E. Haines, "Effects of radiofrequency catheter ablation on regional myocardial blood flow. Possible mechanism for late electrophysiological outcome," Circulation, vol. 89, no. 6, pp. 2667-2672, 1994. 


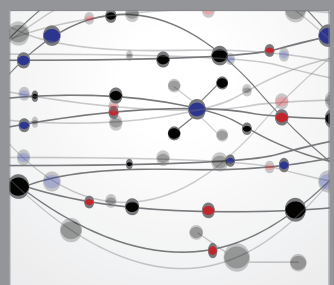

The Scientific World Journal
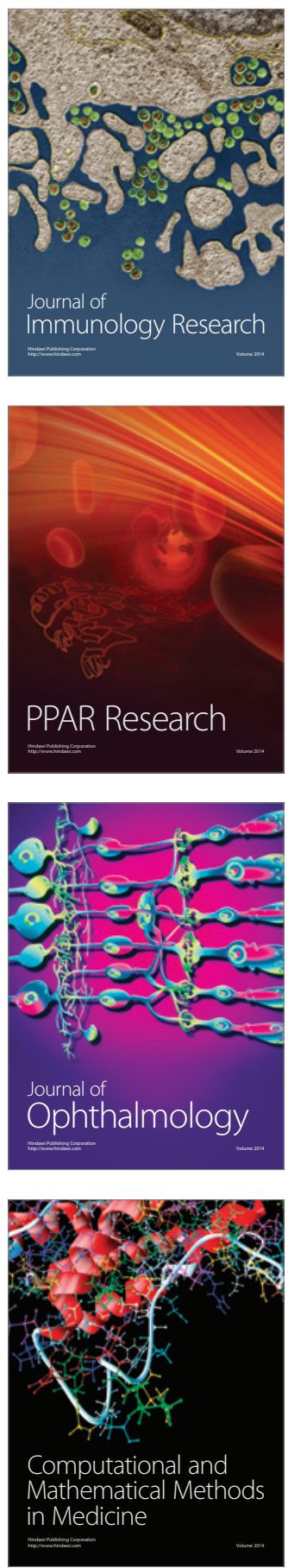

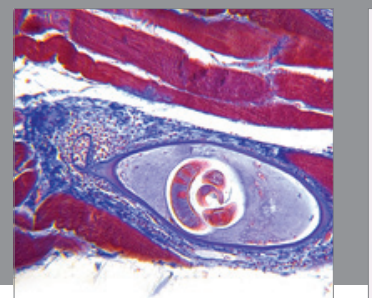

Gastroenterology

Research and Practice
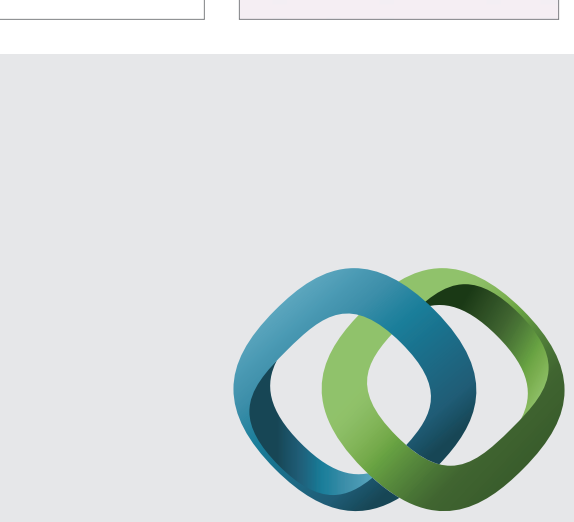

\section{Hindawi}

Submit your manuscripts at

http://www.hindawi.com
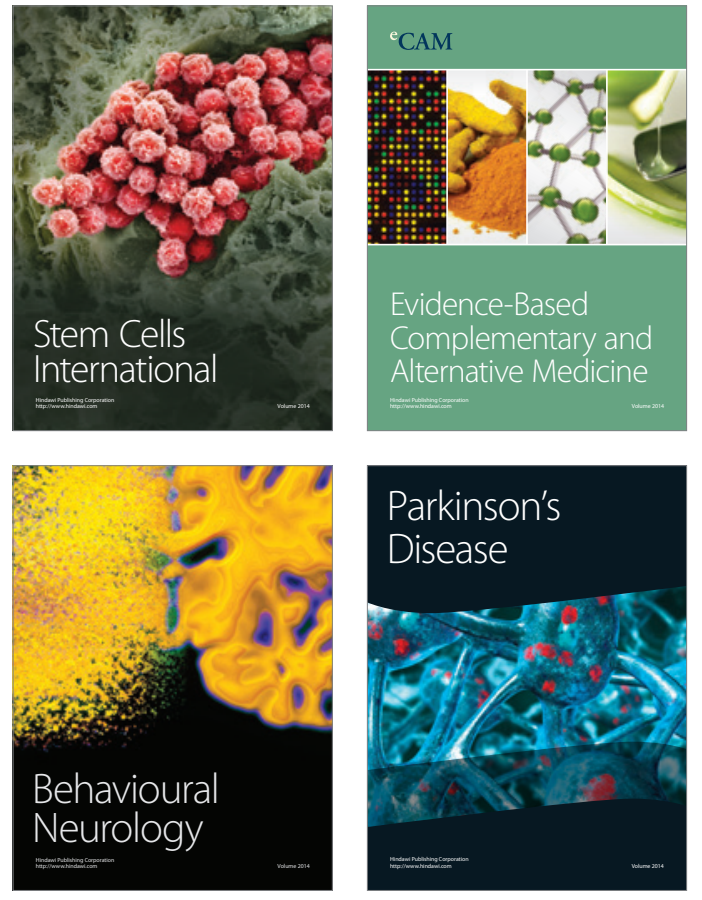
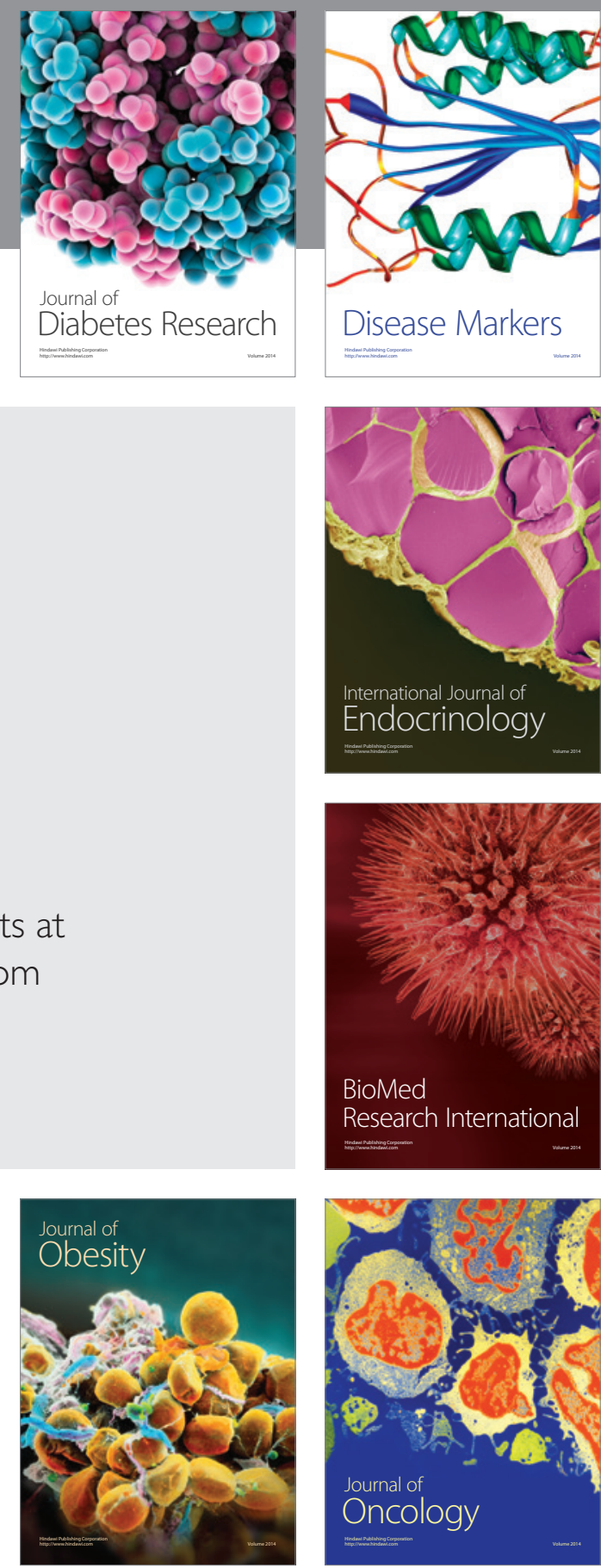

Disease Markers
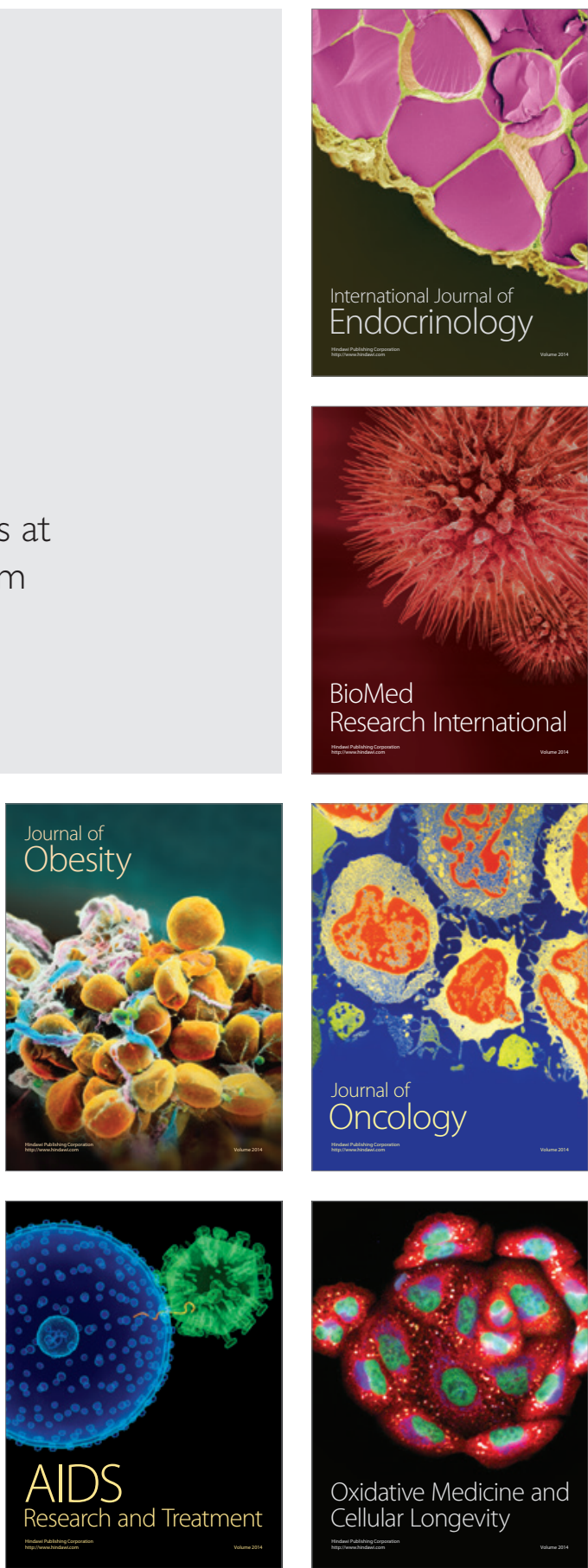